API, стоит выбрать именно эту, ведь она не только способна сделать разработку чат-ботов более функциональной, но и еще у нее очень большой потенциал для дальнейшего совершенствования, так как новые обновления выходят каждый месяц. Также, у этой библиотеки самая подробная и понятная документация.

Таким образом, описанные выше среды и технологии являются одними из наиболее востребованных и подходящих для создания полноценных чат-ботов в среде Telegram.

$$
* * *
$$

1. Джанарсанам, С. Разработка чат-ботов и разговорных интерфейсов / С. Джанарсанам. - Москва : ДМК Пресс, 2019. - 120 c.

2. Как использовать чат-бота в Facebook Messenger для бизнеса. - URL: https://vc .ru/marketing/59475-kakispolzovat-chat-bota-v-facebook-messenger-dlya-biznesa (29.03.2021).

3. Graham, A. Facebook API Developers Guide / A. Graham. - Germany : Apress, 2018 . - 112 p. - ISBN 9781430209690.

4. Сенаторов, А. Telegram. Как запустить канал, привлечь подписчиков и заработать на контенте / А. Сенаторов. - Москва : Альпина Паблишер, 2018. - 160 с.

\title{
Дремов И.С. \\ Нейронные сети как один из методов машинного перевода
}

Оренбургский государственный университет (Россия, Оренбург)

doi: $10.18411 /$ trnio-12-2021-82

\section{Аннотация}

Системы машинного перевода уже довольно давно помогают людям осуществлять переводы множества текстов на различных языках, значительно упрощая жизнь. Есть несколько разных подходов к алгоритмам машинного перевода, но один из самых, на данный момент, интересных - это метод нейронного машинного перевода. Около пяти лет назад произошла практически революция в технологиях машинного перевода, ведь качество переводов улучшилось настолько, что уже стало тяжело отличать текст, переведенный человеком, от текста, переведенного машиной. Произошло это именно благодаря взлетевшей популярности на нейронные сети. Сочетание технологий машинного обучения с привычными методами переводов создало этакие гибридные системы, которые сейчас используются большинством сервисов.

Целью статьи является изучение принципа, по которому нейронные сети производят машинный перевод. Процесс изучения будет включать в себя ознакомление с основными понятиями и типами нейронных сетей.

В результате исследования необходимо установить то, насколько хорош метод нейронного машинного перевода в сравнении переводом, осуществляемым человеком.

Ключевые слова: машинный перевод, нейронная сеть, переводчик.

\section{Abstract}

Machine translation systems have been helping people translate many texts in different languages for quite some time, making life much easier. There are several different approaches to machine translation algorithms, but one of the most interesting at the moment is the neural machine translation method. About five years ago, there was practically a revolution in machine translation technologies, because the quality of translations has improved so much that it has become difficult to distinguish between human-translated text and machine-translated text. This happened precisely due to the skyrocketing popularity of neural networks. The combination of machine learning technologies with familiar translation methods has created the kind of hybrid systems that are now used by most services. 
The aim of the article is to study the principle by which neural networks produce machine translation. The learning process will include familiarization with the basic concepts and types of neural networks.

As a result of the research, it is necessary to establish how well the neural machine translation method is compared to the translation performed by humans.

Keywords: machine translation, neural network, translator.

Нейронный машинный перевод, или сокращенно NMT, представляет собой этакий подраздел машинного перевода, основанный на технологиях машинного обучения и нейронных сетях [1].

В общем смысле, суть перевода с использованием таких технологий заключается в том, что сеть анализирует полученные данные, и на основе этого анализа происходит формирование определенных закономерностей, по которым в последствии строятся предложения. Основывается такой метод на механизме двунаправленных рекуррентных сетей, строясь на матричных вычислениях. Сам процесс обучения требует больших вычислительных мощностей, потому и производится скорее на графических процессорах.

Рекуррентные нейронные сети выбираются именно по причине того, что в отличие от обычных, нерекуррентных сетей, у этих каждое предыдущее состояние нейронной сети - это вход на следующее вычисление, то есть существует влияние предыдущих результатов на последующие. Такой способ позволяет определять шаблоны в последовательности данных, давая возможность предсказывать следующие наиболее вероятные слова, зная только несколько первых из них [2].

Использование двунаправленных рекуррентных сетей обусловлено тем, что для сети очень важно обладать возможностью связывать как предыдущие, так и будущие данные. Это помогает предсказывать недостающее слово «Х» в последовательности, опираясь не только на контекст, расположенный левее слова «Х», но и на то, что должно стоять после слова «Х».

При создании систем нейронного машинного перевода, прежде всего нужно несколько тысяч, а то и сотен тысяч предложений, на основе которых сеть и будет обучаться. Где брать такое количество разработчик решает для себя сам, но как один из вариантов - в интернете сейчас существуют специальные сервисы, в которых собрана огромнейшая база предложений на том или ином языке [3].

Основным языком разработки нейронных сетей считается Руthon и для него уже существует немалое количество библиотек, которые включают в себя все основные функции, необходимые для написания исходного кода.

Принцип, по которому происходит перевод текста из одного языка в другой можно описать как последовательность «кодирование - преобразование - декодирование» входных данных. Каждое слово во входном предложении кодируется как число. Это число сеть переводит в результирующую последовательность чисел, которая представляет собой переведенное целевое предложение [4].

Берется два условных языка - язык «М» и язык «Н». Пишется предложение «С Новым Годом» на языке «М». Данное предложение будет кодироваться на языке «М» числами 0.33, 0.369, 0.963. Соответственно, 0.33 - С, 0.369 - Новым, 0.963 - Годом. Закодированные в числа слова подаются на вход нейронной модели, обученной заранее через анализ сотен тысяч пар предложений языков «М» и «Н». Модель преобразует полученные числа, приводя к новым выходным данным: $0.111,0.125,0.59$. Эти числа декодируются в язык «Н» и выводится результат: «S Novym Shastiem» [5]. Изобразим следующий алгоритм на схеме (рисунок 1). 


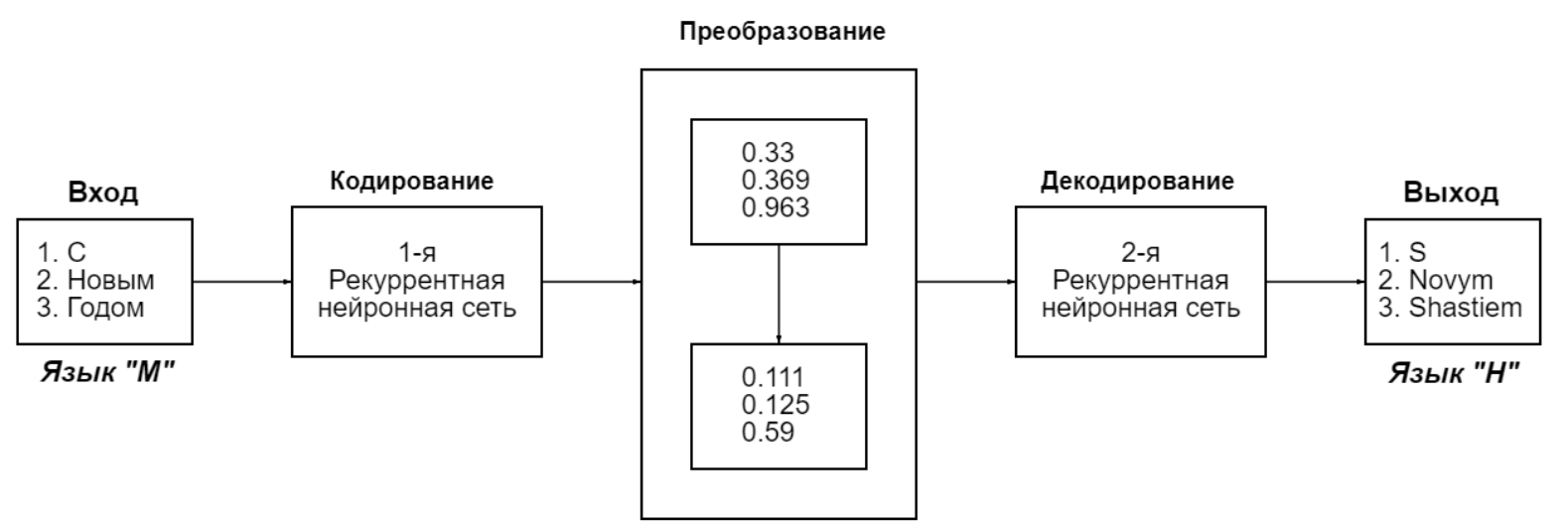

Рисунок 1. Схема алгоритма перевода текста через рекуррентную нейронную сеть

Когда сеть обучается, она проходит через каждую пару предложений с алгоритмом обратного распространения. После каждого прохода, обратное распространение выполняет еще один проход, но уже в другую сторону, тем самым происходит регулировка веса и смещения модели. Таким образом, каждая пара пройденных предложений слегка изменяет нейронную сеть, улучшая результат [6].

Главная проблема сервисов-переводчиков - неумение правильно переводить редкие слова, как в контексте предложений, так и в отдельности.

Как пример, возьмем небольшую часть монолога Александра Андреевича Чацкого главного героя стихотворной комедии «Горе от ума», написанной Александром Грибоедовым, и попробуем перевести ее с использованием сразу трех онлайн сервисов: «Google Translate», «Яндекс Переводчик», «Reverso».

В целом, перевод, изображенный на рисунке 2, действительно можно назвать достойным. Он передает основную мысль стихотворения настолько, насколько это возможно при переводе на другой язык. Однако сервис не смог правильно перевести последнюю строку с устаревшим словом «журьба». Слово, означающее осуждение, он перевел как «рlay» - «игра», и произошло это по причине того, что Google Translate даже не знает о том, что такое слово вообще существует.

А судьи кто? -

За древностию лет

К свободной жизни их вражда непримирима,

Сужденья черпают из забытых газет

Времен Очаковских и покоренья Крыма;

Всегда готовые к журьбе
Who are the judges? -

Over the antiquity of years

Their enmity is irreconcilable to a free life,

Judgments are drawn from forgotten newspapers

The times of the Ochakovskys and the conquest of the Crimea;

Always ready to play

Рисунок 2. Результат перевода через Google

Переводчик от Яндекса (рисунок 3) справился со своей работой гораздо лучше, переведя слово «журьба» именно как «zhurba», тем самым, сохранив смысл предложения. Хотя стоит отметить, что данный сервис является отечественным, а потому ему легче понимать устаревшие русские слова и фразы. Так или иначе, но во время своего обучения сеть, скорее всего, сталкивалась с этим словом.

Асудьи кто?-

За дрееностию лет

Ксвобсднойжизни их вражда непримирима,

Сужденья чеопают из забыть́х газет

Времен Очаковскихи пскоренья Крыма;

Всегда готовые кжурьбе,
And who are the judges? -

For the antiquity of years

Their enmity towards a free life is irreconcilable. Judgments are drawn from forgotten newspapers

The times of Ochakousky and the concuest of the Crimea:

Always ready for zhurba, 
Переводчик Reverso (рисунок 4) справился со своей задачей хуже всех, значительно исказив смысл многих предложений. «За древностию лет» стало звучать просто - «в древности», а фраза о готовности к журьбе вообще превратилась в «always ready to go wild», что скорее переводится как «всегда готов сойти с ума».

А судьи кто? -

За древностию лет

К свободной жизни их вражда непримирима,

Сужденья черпают из забытыіх газет

Времен Очаковских и покоренья Крыма;

Всегда готовые к журьбе,

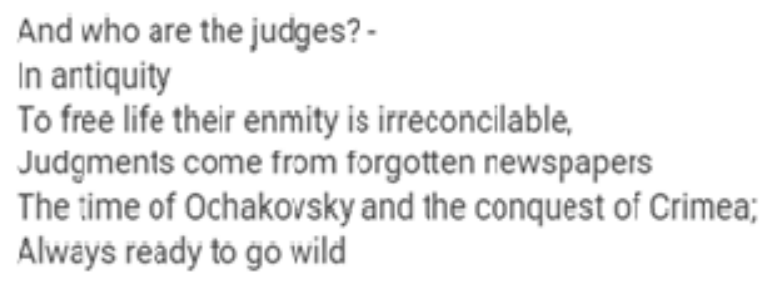

Рисунок 4. Результат перевода через Reverso

Данный пример хорошо демонстрирует, что правильность перевода напрямую зависит от количества слов и предложений, с которыми работает нейронная сеть во время своего обучения, и чем больше ей дать различных вариантов и редких слов, тем лучше она будет работать в будущем.

В настоящий момент нейронные сети очень плотно вошли в нашу жизнь. Их использование в комбинации с привычными методами машинного перевода, несомненно, было правильным решением, и это решение привело к настоящему технологическому скачку. Теперь, благодаря гибридным методам перевода, у каждого человека появилась возможность коммуницировать и изучать другие культуры, не боясь того, что он будет неправильно понят. Это не означает, что смысл в людях-переводчиках отпадает. Нейронные сети еще до конца не научились работать с очень сложными предложениями, устаревшими вариантами написания и многими тонкостями языков. Однако именно внедрение алгоритмов машинного обучения в работу профессиональных переводчиков может повысить качество существующих переводов.

$$
* * *
$$

1. Nabeel T. Alsohybe, Neama Abdulaziz Dahan, Fadl Mutaher Ba-Alwi Machine-Translation History and Evolution: Survey for Arabic-English Translations // Current Journal of Applied Science and Technology. - 2017. S. 1-19.

2. Philipp K. Neural Machine Translation // Center for Speech and Language Processing Department of Computer Science Johns Hopkins University, 2015. S. 133.

3. Shah H., Barber D. Generative Neural Machine Translation // 32nd Conference on Neural Information Processing Systems (NIPS). - 2018. S. 10.

4. Koehn P. Neural Translation Models // Neural Machine Translation by Cambridge University Press. - 2020. S. 125142.

5. TranslateFX [Электронный ресурс]. - Режим доступа: https://www.translatefx.com/blog/what-is-neural-machinetranslation-engine-how-does-it-work (дата обращения: 24.11.2021)

6. Medium.com [Электронный ресурс]. - Режим доступа: https://medium.com/@ppleskov/машинное-обучениеэто-весело-часть-5-51f40fbae4b0 (дата обращения: 23.11.2021).

Кудусова М.И., Алиев А.А., Бакаев Х.А.

Плюсы и минусы дистанционного обучения

ФГБОУ ВО «Чеченский государственный университет им. А.А. Кадырова»

(Россия, Грозньй)

doi: 10.18411/trnio-12-2021-83

\section{Аннотация}

Из-за резкого увеличения числа заразившихся коронавирусом нового типа (covid-19) перед правительством возникла необходимость введения дистанционного обучения. Система образования, долгое время сохранявшая принципы традиционного обучения, перешла в онлайн режим. Учитывая сложившуюся ситуацию, учителя и школьники во многих регионах 